\title{
FELIX: the Detector Interface for the ATLAS Experiment at CERN
}

\author{
Alexander Paramonov ${ }^{1}$ on behalf of the ATLAS TDAQ Collaboration \\ ${ }^{1}$ Argonne National Laboratory, Lemont, Illinois, USA
}

\begin{abstract}
The Front-End Link eXchange (FELIX) system is an interface between the trigger and detector electronics and commodity switched networks for the ATLAS experiment at CERN. In preparation for the LHC Run 3, to start in 2022, the system is being installed to read out the new electromagnetic calorimeter, calorimeter trigger, and muon components being installed as part of the ongoing ATLAS upgrade programme. The detector and trigger electronic systems are largely custom and fully synchronous with respect to the $40.08 \mathrm{MHz}$ clock of the Large Hadron Collider (LHC). The FELIX system uses FPGAs on server-hosted PCIe boards to pass data between custom data links connected to the detector and trigger electronics and host system memory over a PCIe interface then route data to network clients, such as the Software Readout Drivers (SW ROD), via a dedicated software platform running on these machines. The SW RODs build event fragments, buffer data, perform detector-specific processing and provide data for the ATLAS High Level Trigger. The FELIX approach takes advantage of modern FPGAs and commodity computing to reduce the system complexity and effort needed to support data acquisition systems in comparison to previous designs. Future upgrades of the experiment will introduce FELIX to read out all other detector components.
\end{abstract}

\section{Introduction}

The ATLAS experiment at CERN [1] explores collisions of protons and heavy ions from the Large Hadron Collider (LHC) to study physics at the energy frontier. The LHC accelerates bunches of particles to up to $6.5 \mathrm{TeV}$ and collides them every $25 \mathrm{~ns}$ at a combined energy up to $13 \mathrm{TeV}$. It is therefore imperative for the on-detector electronics systems to run synchronously with the LHC clock to digitize the detector signals. The detector electronics systems use custom synchronous serial data links to read out data. The Front-End Link eXchange (FELIX) system serves as an interface between these links and commodity switched networks.

FELIX is installed alongside the existing legacy readout system (ROD and ROS [2] [3]) to readout the electromagnetic (liquid Ar) calorimeters [4], Level-1 calorimeter trigger system [5] and selected muon systems (BIS 7-8 [6] and New Small Wheels [5]) as shown in Fig. 1. The next upgrade stage will take place before LHC Run 4. It will allow ATLAS to 
observe and record collisions at a much higher intensity after the upgrade of the LHC for the High Luminosity program. By Run 4, the FELIX system will be installed to read out all detector components.

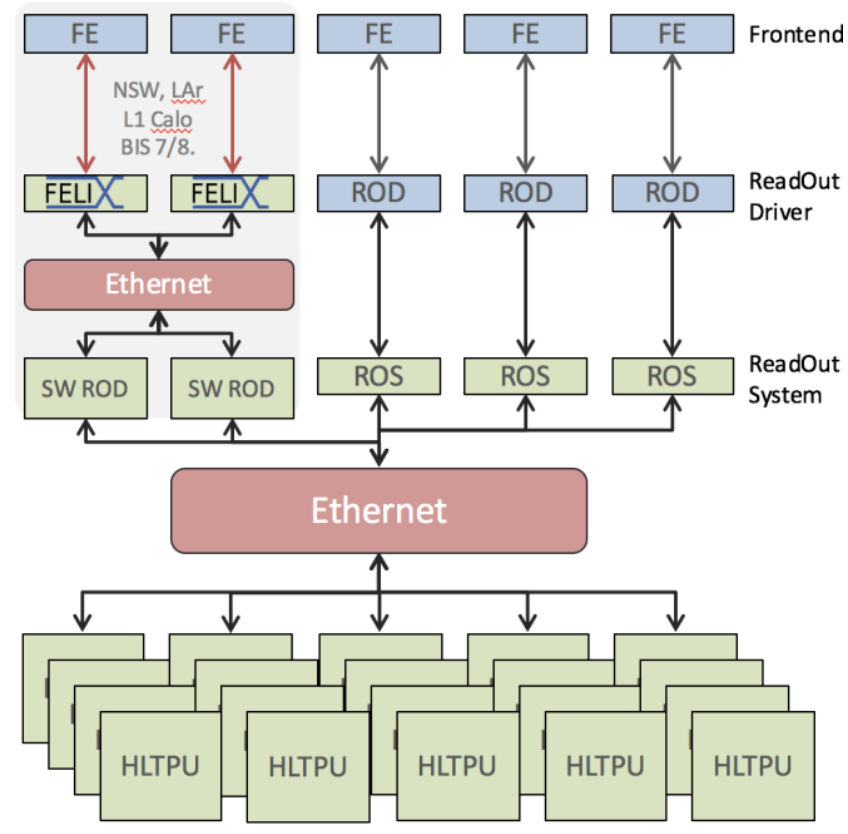

Fig. 1. Present architecture of the ATLAS data acquisition system. FE represents a detector readout system.

To reduce the data rates, the ATLAS trigger system inspects data from every bunch crossing and filters only bunch crossings of interest. Only 1 out of about 40,000 bunch crossings are useful for further processing and stored to disc. The trigger system operates with small and deterministic latency. The trigger passes its decisions to FELIX and it distributes them to the detector readout electronics. The detector readout electronics buffers data while the first level (level-1) hardware trigger system is analyzing it. Currently, the trigger rate is limited to $100 \mathrm{kHz}$, rising to $1 \mathrm{MHz}$ for the high-luminosity programme in Run 4.

The FELIX system consists of commodity servers with PCIe FELIX cards. A FELIX card contains an FPGA interfaced to the PCI express bus and fiber-optic transceivers. One of the optical links receives a serial bitstream from the Timing, Trigger and Control (TTC) system. The TTC system transmits the trigger decisions and LHC-related signals (such as the clock). The other optical links are connected to the detector readout systems, able to operate in a bi-directional manner. The TTC and to-detector links use custom fullysynchronous data transmission protocols. The FPGA allows us to handle data transmissions and to route data between the detector links and the PCIe interface. FELIX software routes data between the FELIX cards and the switched network (e.g. Ethernet).

The FELIX approach was facilitated by the rapid development of commodity switched networks, server CPUs and FPGAs. FELIX reduced the need to develop custom electronics and firmware in comparison to the previous data acquisition architecture. The commercial 
components (e.g network interface cards, servers, network switches) can be easily maintained, upgraded, and customized to meet the needs of the experiment. FELIX's utilization of a switched network also simplifies the software design.

\section{FELIX I/O card}

The FELIX card, also known as FLX-712, is a custom-designed standard PCIe card with a 16-lane PCIe Gen3 interface as shown in Fig. 2. The card features a Xilinx Ultrascale FPGA, XCKU115-FLV-1924, and 8 Avago Minipod transceivers (4 TX and 4 RX). The FPGA has two 8-lane PCIe interfaces, one per super logic region. The card also includes a mezzanine card for the TTC link and a BUSY output. The optical links can operate at speeds up to $12.8 \mathrm{Gbps}$. The BUSY output is connected to the TTC system to make it possible to inhibit the operation of the ATLAS central trigger through a dedicated signal.

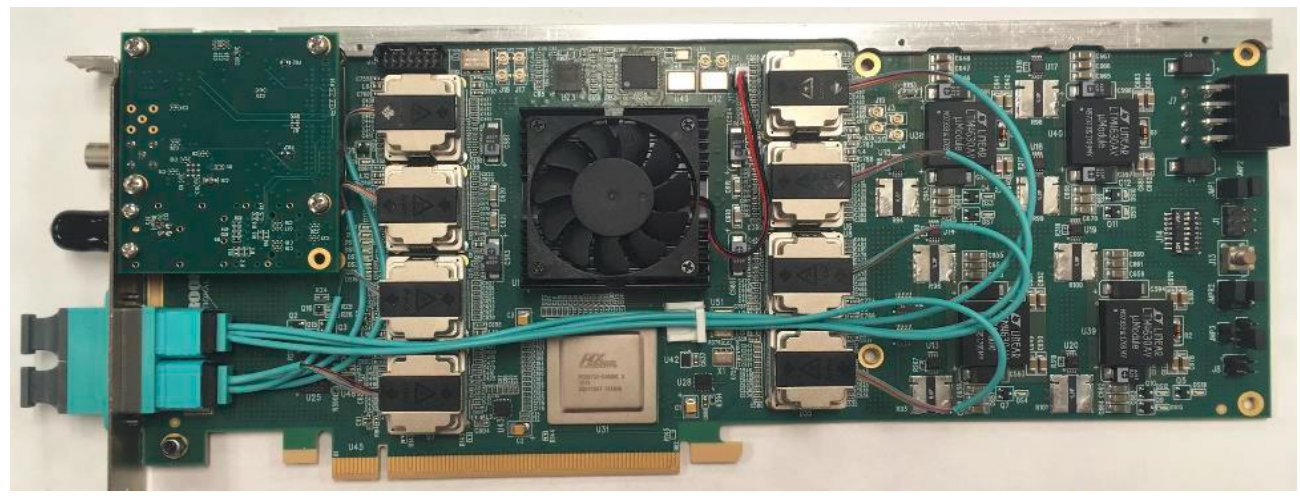

Fig. 2. FELIX PCIe card, FLX712.

A functional diagram for the board components is shown in Fig. 3. The PCIe switch PEX8732 interfaces the 16-lane PCIe slot and the 8-lane FPGA endpoints. The jitter cleaners are needed to provide reference clocks to the Multi-Gigabit GTH Transceivers (MGTs). The ADN2814 IC digitizes the TTC signal as a $160 \mathrm{Mb} / \mathrm{s}$ bitstream and also provides a recovered clock. The FPGA can be programmed via a JTAG connector or PCIe. The $4 \mathrm{TX}$ and $4 \mathrm{RX}$ minipods offer 48 bi-directional links aggregated into two MTP48 couplers. 


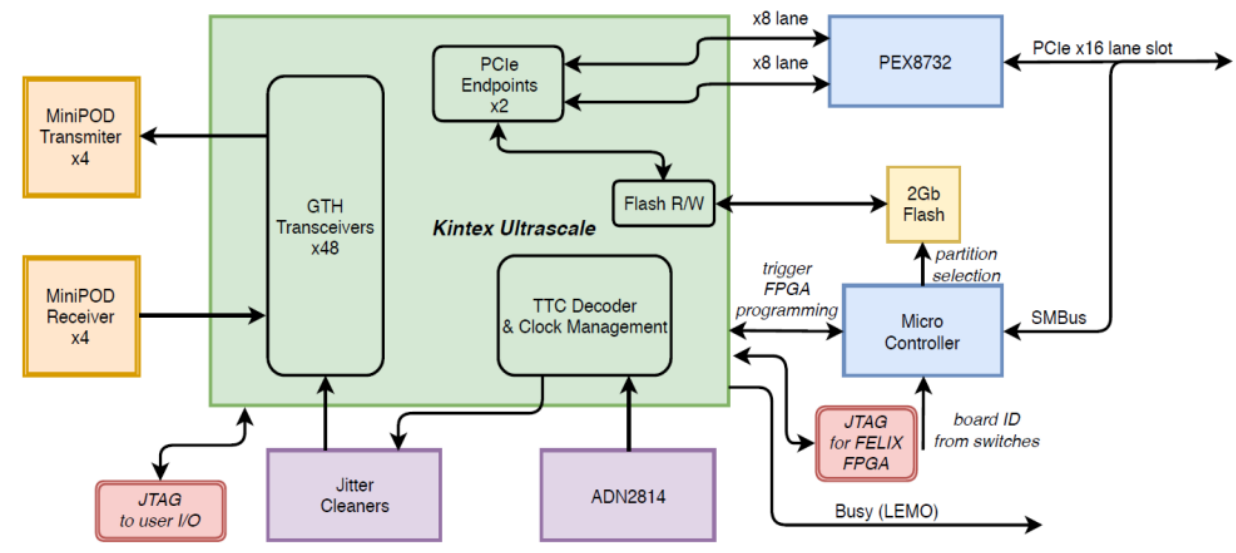

Fig. 3. Functional diagram of FLX-712 components.

The hardware platform has been extensively tested and verified. The throughput of the PCIe interface was measured to be about $100 \mathrm{~Gb} / \mathrm{s}$, in agreement with the specifications. The optical links were characterized for 9.6 and $12.8 \mathrm{~Gb} / \mathrm{s}$ and the bit-error rates were measured to be less than $10^{-15}$ for each of the 48 links. The power consumption of the board is at most $65 \mathrm{~W}$ and the FPGA temperature does not exceed $70 \mathrm{C}$ when housed in the final server configuration to be used on ATLAS Run 3. After completing the verification, 255 FLX-712 boards were produced for the ongoing upgrade and other R\&D needs.

A prototype FELIX board for the Run 4 upgrade has also been designed. It features a Xilinx Versal Prime FPGA and 24 optical data links. The board has a 16-lane PCIe Gen 4 interface. The optical links may support data rates up to $25 \mathrm{~Gb} / \mathrm{s}$. A final decision on the hardware platform won't be made for some time, but ongoing studies with this prototype and its successors will make it possible to closely track technological evolution.

\section{FELIX firmware}

All the serial data links from FELIX to the detector systems use the GBT protocol developed by CERN [8]. The GBT data frame contains 120 bits and the link speed is 4.8 $\mathrm{Gb} / \mathrm{s}$ to make each frame synchronous with the $40.08 \mathrm{MHz}$ clock of the LHC. CERN has also developed a radiation-hard ASIC, GBTx, to aggregate and de-aggregate multiple slower serial links (E-links) into a GBT link. The input high-speed data links from the detector components to FELIX can also use the GBT protocol or a custom $8 \mathrm{~b} / 10 \mathrm{~b}$ encoded protocol operating at $9.6 \mathrm{~Gb} / \mathrm{s}$ called FULL Mode. The MGT transceivers with the GBT encoders and decoders are shown in Fig. 4. 


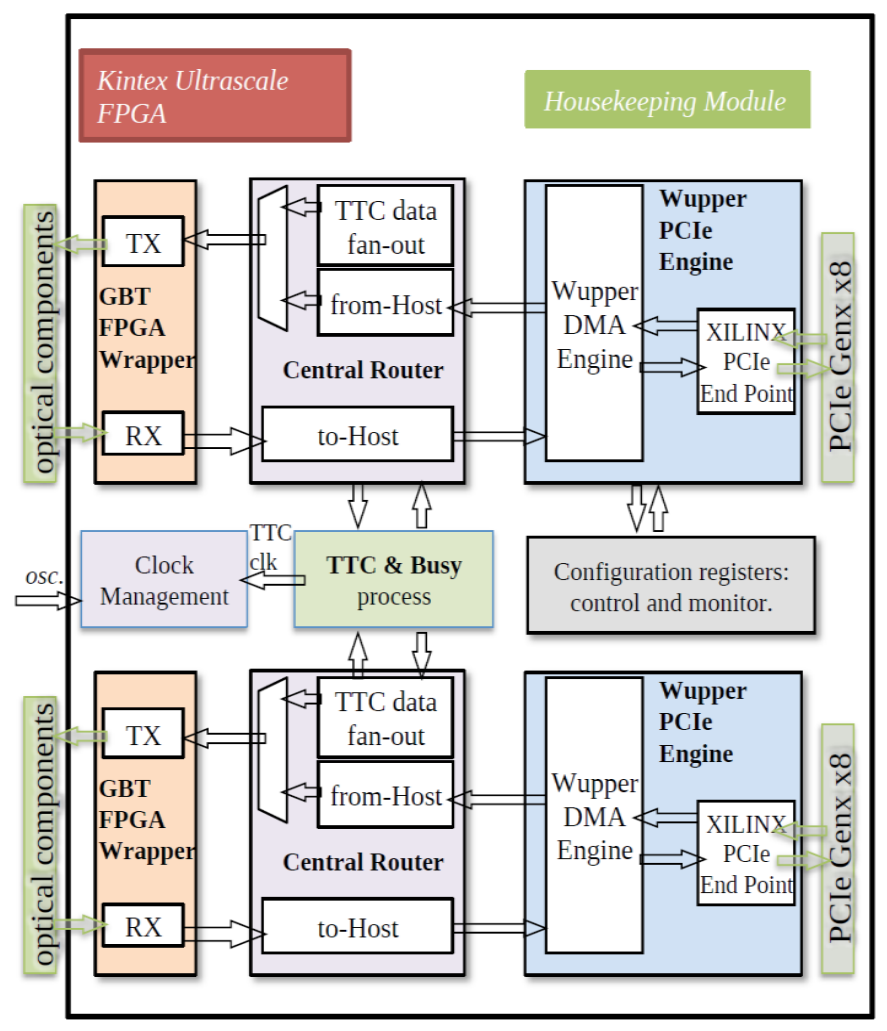

Fig. 4. Functional diagram of FELIX firmware.

The $40.08 \mathrm{MHz}$ LHC clock is recovered from the TTC bitstream and the $160 \mathrm{MHz}$ clock provided by the ADN2818 IC. The $40.08 \mathrm{MHz}$ clock is then passed to the clock management block and to the external jitter cleaner ICs. The GBT wrappers, GTH transceivers, and the central router use the LHC clock and the clock from GTH transceivers (also derived from the LHC clock) to generate signals for the detector electronics. The card can also use a local oscillator to generate a $40.08 \mathrm{MHz}$ clock during stand-alone operation (without the TTC system) outside of the ATLAS experiment.

The TTC system transmits two bits for every LHC bunch crossing. The first bit is the trigger decision and the second bit is used as a serial link for the other signals such as Event Counter Reset, Bunch Counter Reset, and trigger type. The TTC signals are passed to the detector electronics using 8 bits in the GBT data frame. FELIX matches each trigger accept signal to a combination of bunch counter, orbit counter, trigger counter, trigger reset counter and trigger type. All this information is also passed to subscribed processes over the switched network for every accepted bunch crossing (level-1 trigger accept) via the PCIe interface.

The BUSY mechanism is used to prevent data losses. Each front-end system may report a BUSY condition if needed due to internal buffers nearing overflow. The FELIX server may also report BUSY if any internal buffers are about to overflow. The logical OR of the BUSY signals is passed to the BUSY output of the board.

Each of the two PCIe endpoints is serviced by a dedicated firmware engine, called Wupper. Each Wupper engine has a 256-bit wide FIFO transfer data to and from the Central Router module. The FIFOs are connected to Direct Memory Access (DMA) 
interfaces. Each PCIe endpoint supports only 8 PCIe lanes because of a limitation of the Xilinx PCIe Gen3 hard block. The external register map is then connected to each Wupper engine. The registers are used to control and monitor the firmware from the software domain.

The Central Routers pass data between the Wupper blocks and optical links. Some links are serial streams so the data needs to be encoded and decoded accordingly. The Central Router is highly configurable to allow flexible combinations of links and serial protocols for every link.

The firmware has been extensively validated with internal and external data emulators and is undergoing integration with the detector systems: LAr, NSW, BIS 7/8, and calorimeter triggers.

Development of the FELIX firmware for the Run 4 upgrade is progressing rapidly. The future firmware will need to support many more types of detector systems than the present firmware and the lpGBT link protocol in addition to the GBT protocol. Decoders and encoders for pixel and strip modules of the future inner tracker have been designed and are undergoing validation and integration with detector prototypes. FELIX will also need to interact with the new Local Trigger Interface (LTI) system to receive TTC data and to report BUSY states.

\section{FELIX software}

The FELIX software includes drivers, low-level tools, test software and routing software that run on the FELIX server. The low level tools are needed to control and configure the FPGAs. The FELIX cards are accessed and controlled with drivers known as flx and cmem_rcc. The flx driver provides access to the register map (and therefore control of the card). The cmem_rcc driver allows applications to allocate large contiguous buffers in the server memory for high-bandwidth access via DMA. The routing tool, known as felix-star, distributes data between the buffers and subscribed applications via the network as shown in Fig. 5. felix-star uses a single-thread asynchronous event loop architecture. These events have non-blocking callback functions to process interrupts from the FL712, send and receive data and handle file description. The subscribed applications use a library known as NetIO-next to communicate with felix-star. NetIO-next can use POSIX sockets or libfabric. RDMA over Converged Ethernet (RoCE) reduces the number of data copies to transfer data, avoids context-switching by bypassing the kernel and does not use either source or client CPU. The performance of felix-star has been tested and verified for the trigger rates up to $200 \mathrm{kHz}$, with the rate limited by network bandwidth (currently $100 \mathrm{GbE}$ ). The level or performance exceeds the requirements for Run 3 by a factor of 2 . 


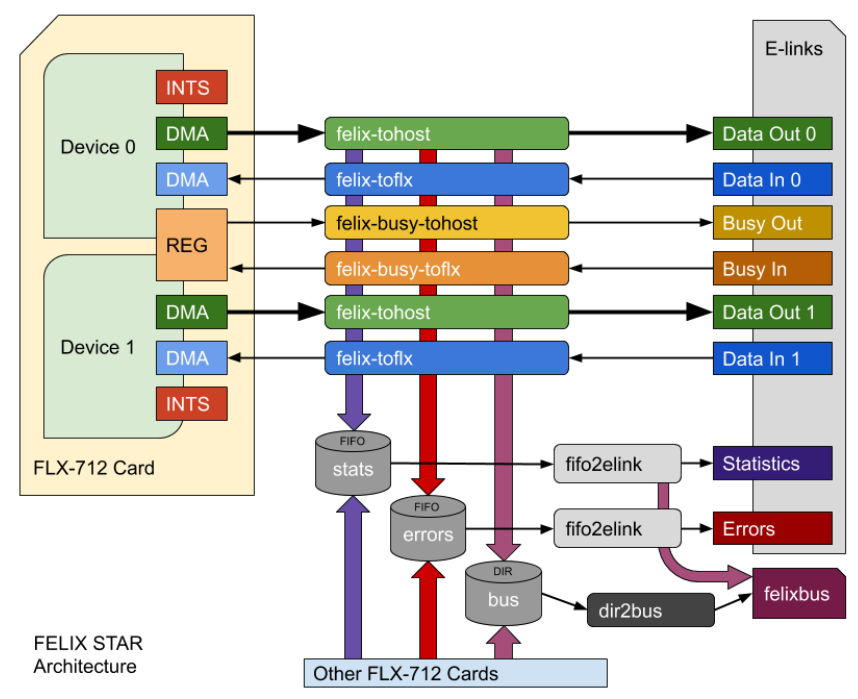

Fig. 5. Architecture of felix-star data routing tool.

A Software ReadOut Driver (SW ROD) [9] receives data from a few FELIX servers to aggregate and format the data to serve it to the High Level Trigger (HLT). The incoming data fragments are aggregated into a group (event) for each accepted bunch crossing. The SW ROD application is a customizable framework that can support detector-specific algorithms for event building and processing. The SW ROD needs to handle data from particle collisions and detector-specific activities such as calibration runs. The SW ROD application is designed as a number of independent components with simple interfaces to interact between each other. The components receive data fragments, build events and distribute the data to the HLT or other consumers.

The SW ROD architecture is optimized for high throughput processing. It is capable of event building at rates exceeding $100 \mathrm{kHz}$ while the input packet rate is up to $120 \mathrm{MHz}$. The performance was limited by the network bandwidth, with three processing threads to handle the data volume. SW ROD is being developed further to build events at a $1 \mathrm{MHz}$ rate for Run 4 . The memory management was found to be a bottleneck for $1 \mathrm{MHz}$ data processing and a new memory management approach was introduced. With this improvement, the SW ROD was shown to already be able to process a $1 \mathrm{MHz}$ event rate for selected link configurations.

\section{Conclusions and Outlook}

The FELIX data acquisition system for the ATLAS experiment bridges the custom fully synchronous detector electronics and commodity computing. FELIX is being installed and commissioned for the ongoing upgrade to start operation in 2022. About 100 FELIX cards are installed into 60 servers and another 20 servers are set up to run SW ROD. FELIX hardware, firmware, and software are evolving rapidly to meet the future needs and to readout all components of the ATLAS experiment after the next upgrade at a 10 times higher trigger rate.

\section{Acknowledgements}


Argonne National Laboratory's work was supported by the U.S. Department of Energy, Office of High Energy Physics, under contract DE-AC02-06CH11357.

\section{References}

[1] ATLAS Collaboration, "The ATLAS Experiment at the CERN Large Hadron Collinder," Jinst, vol. 3, p. S08003, 2008.

[2] A. Gabrielli, "Commissioning of ROD boards for the entire ATLAS pixel detector," Journal of Instrumentation, vol. 13, no. 09, pp. T09 009-T09 009, 2018.

[3] ATLAS TDAQ Collaboration, "The ATLAS Data Acquisition and High Level Triggersystem," JINST, vol. 11, no 6, p. P006008, 2016.

[4] M. Aleksa and others, "ATLAS Liquid Argon Calorimeter Phase-I Upgrade Technical Design Report," CERN-LHCC-2013-017, 2013.

[5] ATLAS Collaboration, "Technical Design Report for Phase-I Upgrade of the ATLAS TDAQ System," CERN-LHCC-2013-018, 2013.

[6] L. Massa, "The BIS78 Resistive Plate Chambers upgrade of the ATLAS Muon Spectrometer for the LHC Run-3," JINST, vol. 15, no. 10, pp. C10026-C10026, 2020.

[7] B. Stelzer, "The New Small Wheel Upgrade Project of the ATLAS Experiment," Nuclear and Particle Physics Proceedings, vol. 273275, pp./ 1160-1165, 2016.

[8] P. Moreira, R. Ballabriga, S. Baron, S. Bonacini, O. Cobanoglu, F. Faccio, T. Fedorov, R. Francisco, P. Gui, P. Hartin, K. Kloukinas, X. Llopart, A. Marchioro, C. Paillard, N. Pinilla, K. Wyllie and B. Wu, "The GBT Project," Proc. Topical Workshop on Electronics for Particle Physics, pp. 342-346, 2009.

[9] S. Kolos, "New software based readout driver for the ATLAS experiment," presented at the 22nd IEEE Real Time Conference, Virtal, Vietnam, 2020. 